\title{
Atomic-Scale Friction Studies on Single-Crystal Gallium Arsenide Using Atomic Force Microscope and Molecular Dynamics Simulation
}

\author{
Pengfei Fan $^{1} \cdot$ Saurav Goel ${ }^{2,3,4}\left(\mathbb{C}^{\circ} \cdot\right.$ Xichun Luo $^{1} \cdot$ Hari M. Upadhyaya ${ }^{2}$
}

Received: 22 February 2021 / Revised: 12 May 2021 / Accepted: 8 June 2021 / Published online: 10 July 2021

(c) The Author(s) 2021

\begin{abstract}
This paper provides a fresh perspective and new insights into nanoscale friction by investigating it through molecular dynamics (MD) simulation and atomic force microscope (AFM) nanoscratch experiments. This work considered gallium arsenide, an important III-V direct bandgap semiconductor material residing in the zincblende structure, as a reference sample material due to its growing usage in $5 \mathrm{G}$ communication devices. In the simulations, the scratch depth was tested as a variable in the fine range of $0.5-3 \mathrm{~nm}$ to understand the behavior of material removal and to gain insights into the nanoscale friction. Scratch force, normal force, and average cutting forces were extracted from the simulation to obtain two scalar quantities, namely, the scratch cutting energy (defined as the work performed to remove a unit volume of material) and the kinetic coefficient of friction (defined as the force ratio). A strong size effect was observed for scratch depths below $2 \mathrm{~nm}$ from the MD simulations and about $15 \mathrm{~nm}$ from the AFM experiments. A strong quantitative corroboration was obtained between the specific scratch energy determined by the MD simulations and the AFM experiments, and more qualitative corroboration was derived for the pile-up and the kinetic coefficient of friction. This conclusion suggests that the specific scratch energy is insensitive to the tool geometry and the scratch speed used in this investigation. However, the pile-up and kinetic coefficient of friction are dependent on the geometry of the tool tip.
\end{abstract}

Keywords AFM nanoscratching $\cdot$ Molecular dynamic (MD) simulation $\cdot$ Single-crystal gallium arsenide $\cdot$ Friction

\section{Introduction}

Gallium arsenide (GaAs) is one of the hard-brittle materials with desirable characteristics, such as hightemperature resistance [1], large bandgap [2], and high electronic mobility [3] making it superior to silicon as a semiconductor material. Fabrication of nanosize features and shapes on GaAs can be suitably performed by using

Saurav Goel

goels@1sbu.ac.uk

Xichun Luo

xichun.luo@strath.ac.uk

1 Centre for Precision Manufacturing, DMEM, University of Strathclyde, Glasgow, UK

2 School of Engineering, London South Bank University, 103 Borough Road, London SE1 0AA, UK

3 School of Aerospace, Transport and Manufacturing, Cranfield University, Bedfordshire MK43 0AL, UK

4 Department of Mechanical Engineering, Shiv Nadar University, Gautam Buddh Nagar 201314, India atomic force microscopy (AFM) tip-based nanoscratching. As a form of contact mode machining, AFM achieves a much higher material removal rate than focused ion beam machining. However, AFM-based nanoscratching has gained pace only since the development of AFM, dating back to the 1990s. Hence, very limited literature exists around AFM-based investigation on a single-crystal material GaAs. An early work explored the possibility of directly patterning the furrows on a single-crystal GaAs surface $[4,5]$ and demonstrated its feasibility. 5G communication devices are expected to gain extreme popularity soon. Therefore, the mechanism of material removal, the origins of plasticity, and most importantly the kinetic coefficient of friction (COF) GaAs offers to a diamond tool during nanoscratching need to be understood. Obtaining the kinetic COF is an important element in nanotribology, because many analytical equations that are used to obtain insights into wear processes require the value of the $\mathrm{COF}$ a priori. However, previously reported values of $\mathrm{COF}$, except those in the work of Komanduri et al. [6], exhibited a wide range, varying from extremely 
low $(\sim 0.005)$ to intermediate values $(0.13)$ to high values (1.2 and 5). Moreover, previous works have investigated nanoscale friction on aluminum, which is a facecentered cubic ductile metal. Hence, some doubt exists as to whether the same conclusions will still apply to a zincblende lattice structure material such as GaAs. A survey of materials in the literature shows a wide range of values for the COF depending on the material's crystal structure, as listed in Table 1.

A similar investigation on a brittle-hard semiconductor material such as GaAs is necessary to ensure whether the nature of atomic-scale friction in this material is similar or different from that of the materials listed in Table 1. An open-ended question is whether GaAs behaves in the same way as aluminum in terms of the nanoscale scratching size effect and whether the force ratio and specific scratching energy in GaAs scale with the scratch depth or remain unchanged. These questions are the primary motivation behind this work. According to the literature review, many molecular dynamics (MD) studies were published during the last decade and further studies on nanomachining of GaAs are emerging. These studies shed light on aspects of crack formation [15] during singlepoint diamond turning (SPDT), plastic deformation of GaAs [16], and the material removal mechanism during chemo-mechanical polishing [17]. However, these studies did not address aspects of the size effect observed in GaAs much like the other brittle materials, and they also did not clarify whether the kinetic COF is a sufficiently robust indicator to compare simulations and experiments, especially in this era of the digital twin. A well-planned experiment and MD simulation methodology were developed by undertaking a thorough investigation to obtain various insights that are relevant to the cost-effective nanomanufacturing of $\mathrm{GaAs}$; this effort is considered a novel one.

\section{Research Methodology}

\subsection{Simulation Methodology}

MD simulations were performed via an open-source code, namely, large-scale atomic/molecular massively parallel simulator (LAMMPS) [18]. The three-dimensional (3D) nanoscratching model that is used to emulate the AFM nanoscratching developed in this work is shown in Fig. 1. The atoms of the single-crystal GaAs workpiece are divided into three zones, namely, the Newton atoms, thermostat atoms, and boundary atoms, following the previously published literature on this topic [19-22]. The boundary atoms were kept fixed to maintain the symmetry of the initial lattice during MD simulation. The thermostat atoms were set to follow the Berendsen thermostatic dynamics [23] to maintain the thermodynamics of the system by using them as a heat dissipate or in place of a coolant, which was not considered in the simulation model. Newton atoms were allowed to follow the Newtonian (NVE) dynamics of LAMMPS. A pyramid-shaped diamond tool was modeled to mimic the AFM tip, which contained nearly 17,132 atoms. The AFM tip was modeled as a rigid non-deformable body because the cutting distance considered in this work was too small, and the intention was not to study the tool wear, which is an aspect that was already reported previously [24]. The diamond tip was prescribed a velocity of $10 \mathrm{~m} / \mathrm{s}$ into the GaAs workpiece, which was oriented on the (110) plane and cutting in the $<001>$ direction, maintaining the $z$ in the $<-110>$ direction. This plane was chosen for cutting because previous investigations showed that the (110) plane is an easy cutting direction and a favorable orientation for cutting materials such as diamond and zincblende $\mathrm{SiC}$ [7]. However, the preferred cutting direction for silicon is the (111) orientation. The prismatic shape of the diamond tip was oriented in such a way that one of its edges was parallel to the $z$ plane of the workpiece in the scratch direction. The
Table 1 COF in various materials

\begin{tabular}{llll}
\hline Material & Lattice structure and lattice plane & Method and counter material & COF \\
\hline Silicon carbide [7] & Zincblende (001) & Nanoscratching with diamond & 0.70 \\
& Zincblende (110) & & 0.64 \\
& Zincblende (111) & & 0.66 \\
Titanium [8] & Body-centered cubic (001) & Nanoscratching with silicon nitride & 0.76 \\
Tantalum [9] & Body-centered cubic (001) & Nanoscratching with diamond & 0.68 \\
Nickel [10] & Face-centered cubic (100) & Nanoscratching with nickel & 0.60 \\
Copper [11] & Face-centered cubic (110) & Nanoscratching with copper & 0.27 \\
& Face-centered cubic (100) & & 0.46 \\
PMMA [12] & - & Nanoscratching with steel ball & 0.50 \\
Glass [13] & Amorphous solid & Nanoscratching with diamond & 0.12 \\
Graphene [14] & Hexagonal & Nanoscratching with diamond & 0.22 \\
\hline
\end{tabular}


Fig. 1 MD simulation model of nanoscratching of GaAs using a rigid AFM-based diamond tip. Detail $A$ refers to a region of atoms used to obtain the scalar value of von Mises stress in the cutting region

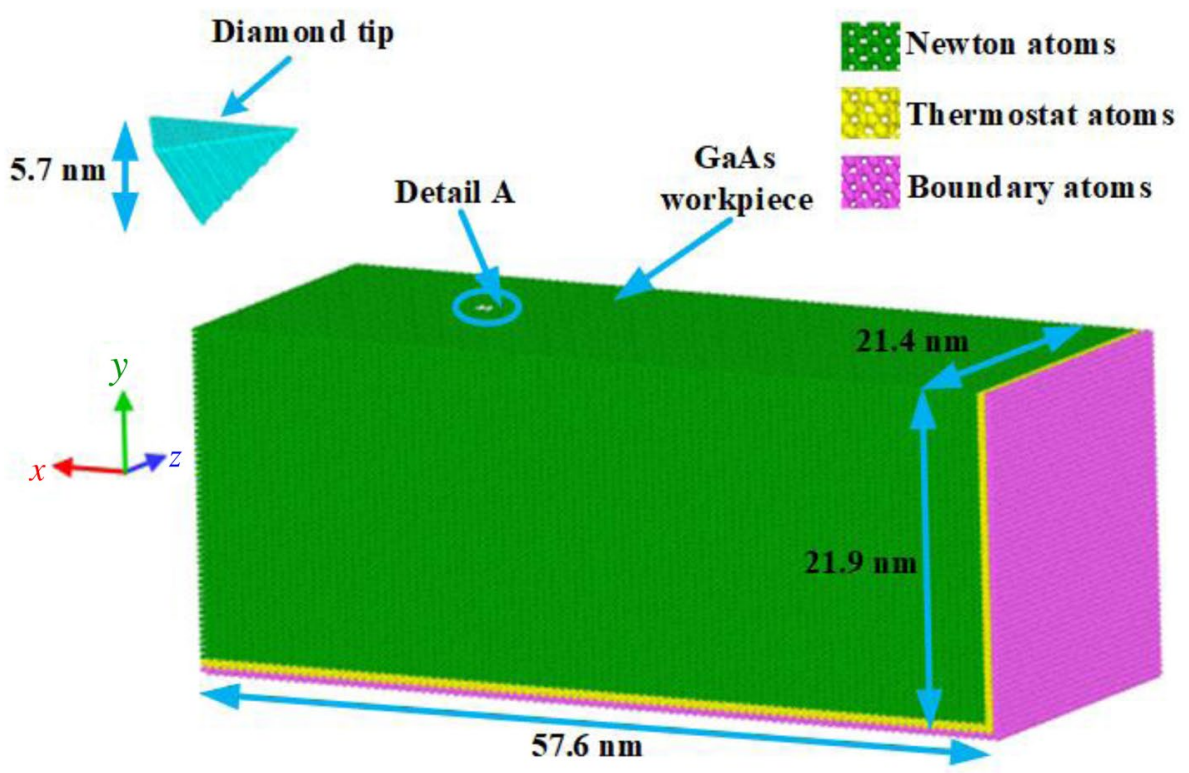

model was initially equilibrated for about 20 ps by using the Nose-Hoover method [25]. A 3D stress unit region (containing nearly 44 atoms of GaAs) was chosen to monitor the scalar value of stress acting on the workpiece during the nanoscratching process.

An important consideration in an MD simulation is to describe the material as realistically possible [26]. For this reason, this investigation used a three-body analytical bond order potential (ABOP) $[27,28]$ that was fully parameterized to describe the zincblende phase of $\mathrm{Ga}, \mathrm{As}$, and $\mathrm{C}$ atoms. The cross interactions between $\mathrm{C}-\mathrm{Ga}$ and $\mathrm{C}-\mathrm{As}$ atoms were described using the Ziegler-Biersack-Littmark (ZBL) potential function [29]. Further details about the ZBL potential are available on the LAMMPS webpage [30]. To perform image rendering and improve data visualization, another software program called Open Visualization Tool
[31] was used. For repeatability, the MD simulation parameters used in this work are described and demonstrated in Table 2. The simulations were performed on the UK's [32] ARCHER2 High-Performance Computer, which has about 12,800 cores (each node on ARCHER2 has about 128 cores), and each simulation finished within $5 \mathrm{~h}$.

\subsection{Experimental Setup}

The nanoscratching experiments were performed on the (110)-oriented GaAs surface by using a triangular pyramid diamond tip (Micro Star Technologies Ltd. [US]) on a commercial AFM platform with a Nanoman module (Dimension Icon, Bruker Corporation, Germany). The nanoscratching feed direction was kept perpendicular to the cantilever of the diamond tip to prevent it from bending due to the scratching

Table 2 Parameters in MD simulations

\begin{tabular}{ll}
\hline GaAs lattice constant & $5.65 \AA$ (zincblende lattice structure) \\
Diamond lattice constant & $3.56 \AA$ (diamond cubic lattice structure) \\
Workpiece material & $\begin{array}{l}\text { Single-crystal GaAs with total of about } 1,190,970 \text { atoms } \\
57.6 \mathrm{~nm} \times 21.9 \mathrm{~nm} \times 21.4 \mathrm{~nm} \text { in the } x(001), y(110), \text { and } z(-110) \\
\text { directions }\end{array}$ \\
$\begin{array}{ll}\text { Diamond tip (rigid) with a total of about } 17,132 \text { carbon atoms } \\
\text { Scratch tool }\end{array}$ & $\begin{array}{l}0.5,1,1.5,2,2.5, \text { and } 3 \mathrm{~nm} \\
\text { Scratch depth (variable) }\end{array}$ \\
$\begin{array}{l}\text { Scratch distance } \\
\text { Scratch velocity of the diamond tip }\end{array}$ & $10 \mathrm{~m} / \mathrm{s}$ \\
Crystallographic plane of GaAs workpiece and scratching direction & $<001>(110)$ \\
Initial temperature of the workpiece & $300 \mathrm{~K}$ \\
Boundary conditions & Periodic, shrink-wrapped, and periodic along the $x, y$, and $z$ direc- \\
Time step of MD calculation & $1 \mathrm{fs}$
\end{tabular}


load [33]. A schematic of the nanoscratching methodology that was followed during the experiments is shown in Fig. 2. The diamond tip was maneuvered into the workpiece at a velocity of $5 \mu \mathrm{m} / \mathrm{s}$ for each scratch under $0.05-0.4 \mathrm{~V}$ applied voltage. The preset normal load $\left(F_{N}\right)$ from this information can be estimated as $F_{N}=$ Voltage $\times K_{N} \times$ sensitivity, where $K_{N}$ refers to the spring constant of the cantilever of the diamond tip, which was $200 \mathrm{~N} / \mathrm{m}$ as specified by the manufacturer. The sensitivity was measured to be $627.5 \mathrm{~nm} / \mathrm{V}$ by pressing the diamond tip on a sapphire specimen surface. Therefore, the normal load $\left(F_{N}\right)$ was estimated to be in the range of $6.28-50.20 \mu \mathrm{N}$ for the applied voltage of $0.05-0.4 \mathrm{~V}$, respectively. The normal load was set small enough to ensure that the material removal during the nanoscratching occurs in the ductile regime and cracking in the wafer can be avoided. After nanoscratching, a sharp silicon tip was employed to measure the topography of all the nanoscratched surfaces.

\section{Results and Discussion}

\subsection{Surface Topography of the Nanoscratches}

The surface topography of various surfaces obtained at scratch depths of $0.5,1,1.5,2,2.5$, and $3 \mathrm{~nm}$ is shown in Fig. 3. The atoms in Fig. 3 are colored by a height map by keeping the bottom of the scratch depth as the $0 \mathrm{~nm}$ datum and by measuring the top pile-up height in increasing order. Thus, for instance, for a scratch depth of $0.5 \mathrm{~nm}$, the bottom of the scratch surface has a $0-\mathrm{nm}$ datum, and the theoretical reference surface would be $0.5 \mathrm{~nm}$ above the datum, which means that if the height label is $0.8 \mathrm{~nm}$, then the pile-up height is $0.3 \mathrm{~nm}$ above the surface. Figure 3 shows that at shallower scratch depths, the pile-up scales linearly as the scratch depth increases. Moreover, the pile-up occurred at
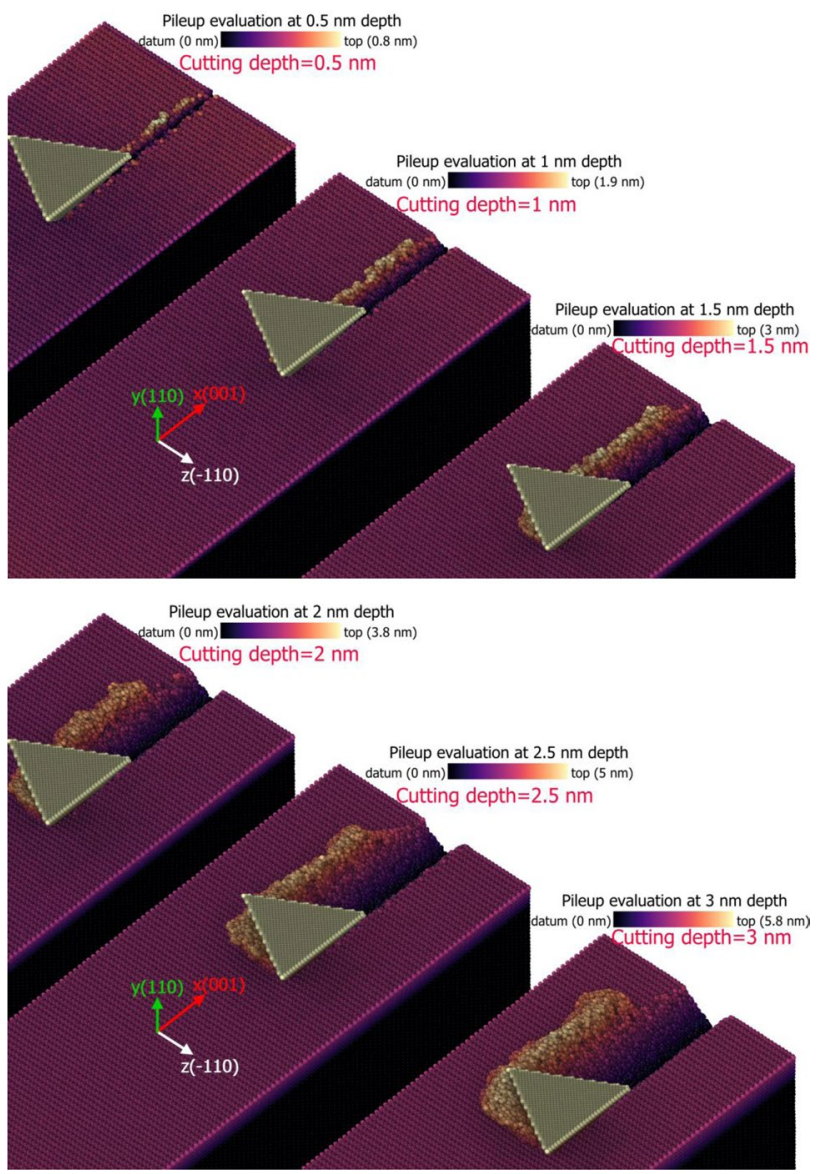

Fig. 3 Assessment of the pile-up, flow of cutting chips, and nature of the chip flow during the material removal observed from the MD simulations. Colors represent the height map of the atoms in the vertical $Y$ direction

only one prismatic side of the tool tip because of the oblique cutting angle presented by the AFM tool tip.
Fig. 2 Schematic of AFM tip-based nanoscratching on single-crystal GaAs

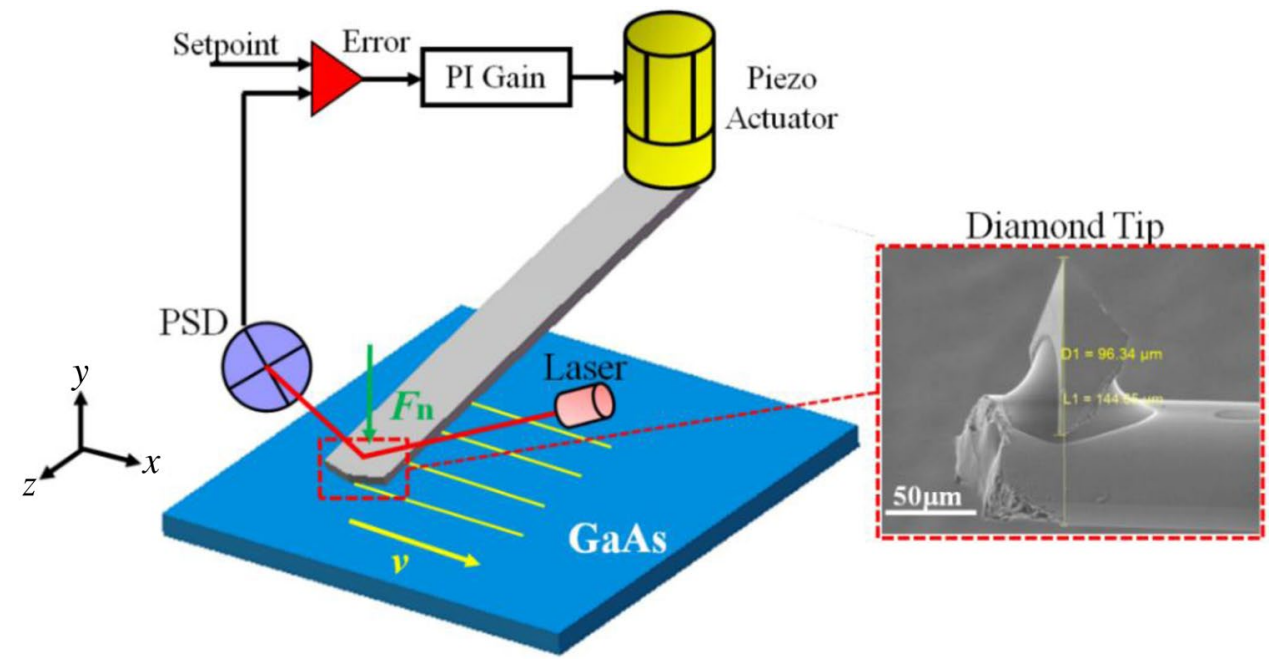


The experimental AFM topography of the measured scratches is shown in Fig. 4. A total of eight scratches were made in the AFM at various loads, and their indicative scratch depths are plotted in the cross section in Fig. 4b. At shallow depths, the pile-up occurred on one side, similar to the condition observed during the MD simulation. The pile-up at shallow depths also scales linearly with increasing depth.

\subsection{Scratch Force, COF, and Specific Scratching Energy}

The evolution of the (i) friction force $(F x)$, which is the force acting in the direction of scratching, and (ii) the normal force $(F y)$, which is the force acting in the direction perpendicular to the tool movement, is shown in Fig. 5. The resultant force described as the square sum of these forces was estimated using the formula $\left(F r=\sqrt[2]{F_{x}^{2}+F_{y}^{2}}\right)$.

In the early stages of contact, the force increases from 0 to a certain value (in this regime, compression of the workpiece material is dominant); this regime is also referred to as an unsteady cutting state. Thereafter, once the material starts to flow, the shear stress dominates and the force value becomes saturated. This regime is referred to as steady-state cutting. With the increasing scratch depth, the normal force becomes greater than the friction force, thereby indicating the reduced kinetic COF.

Also, the magnitude of the $F y$ was larger than that of $F x$, especially at a greater scratch depth, because of the active negative rake angle presented by the triangular pyramid tip similar to that of a cutting tool used in the machining of brittle materials like silicon during SPDT [34-36] or the grinding process [37]. The nature of forces increases linearly as the cutting depth increases. The resultant force multiplied by the scratching distance and divided by the volume of the removed material gives a scalar quantity called specific scratching energy, which is considered independent of the size of the tool and is representative of the material's resistance to cutting at various depths. The variation in the estimated average cutting force ( $\mathrm{Fr}$ ) and the specific scratch energy at various depth of scratches obtained from the MD simulations are shown in Figs. 6 and 7. The nature of this variation follows an identical trend to that of the force ratio of the kinetic COF (defined by $F x / F y$ ) shown in Fig. 8. The specific scratching energy was as high as $75 \mathrm{GPa}$ with a kinetic COF approaching a value of 0.87 at a shallow scratch depth of $0.5 \mathrm{~nm}$. This value becomes more saturated at about $17 \mathrm{GPa}$ and 0.62 at higher scratch depths of $3 \mathrm{~nm}$. The steepness of this variation was mild for scratch depths between 1.5 and $3 \mathrm{~nm}$ but had a bigger slope of $70 \mathrm{GPa} / \mathrm{nm}$ within the scratch depth of 0.5 to $1 \mathrm{~nm}$. The nature and trend of the force ratio and specific scratching energy are reminiscent of the previously reported work on aluminum discussed in the introduction and hence clearly indicated a strong size effect in GaAs much like aluminum.

Figure 9 shows the corresponding AFM experimental results obtained for various scratches performed from 2 to $30 \mathrm{~nm}$ scratch depths. Not only did the AFM experiments in common with the MD simulations show an increasing magnitude of the cutting forces with the scratch depth, but the kinetic $\mathrm{COF}$ also indicated a strong size effect. The ratio
Fig. 4 Experimental AFM image a surface morphology of various nanogrooves and $\mathbf{b}$ height map of various scratches
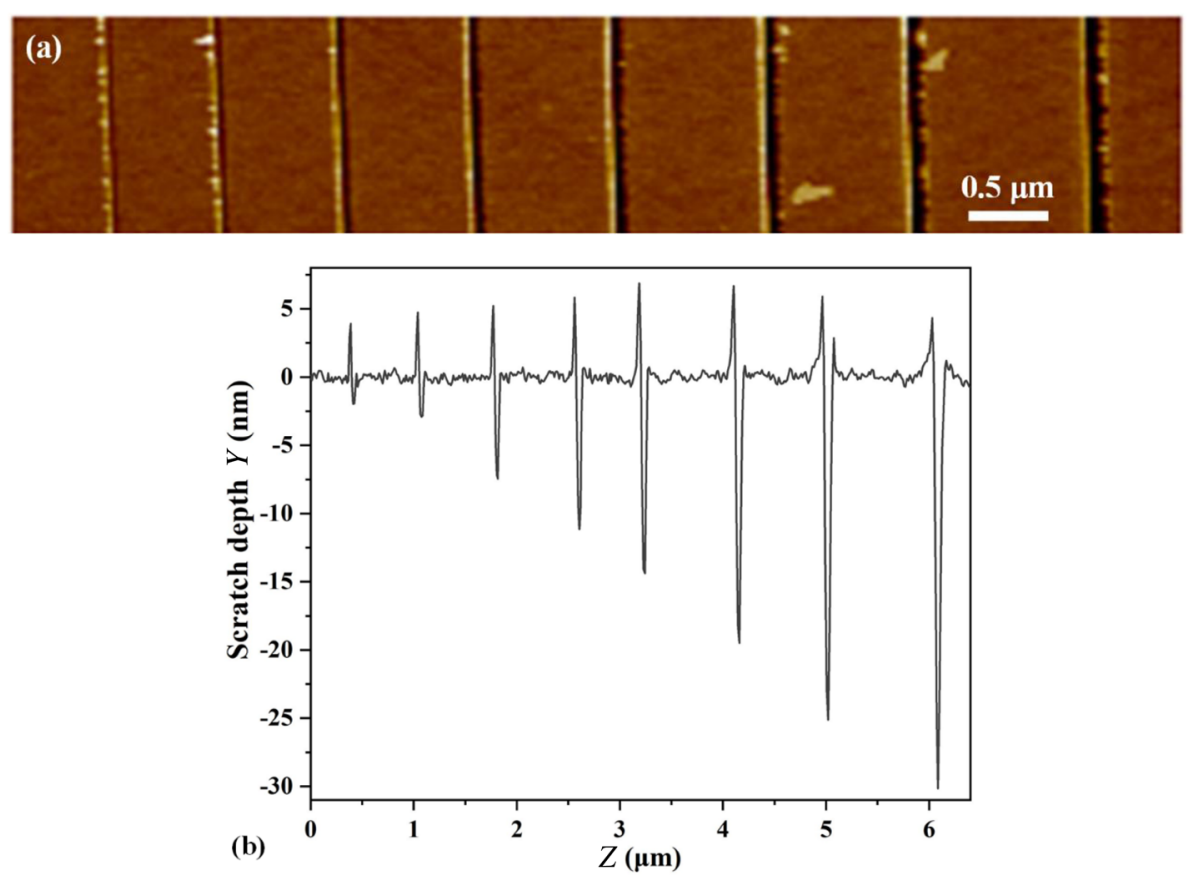

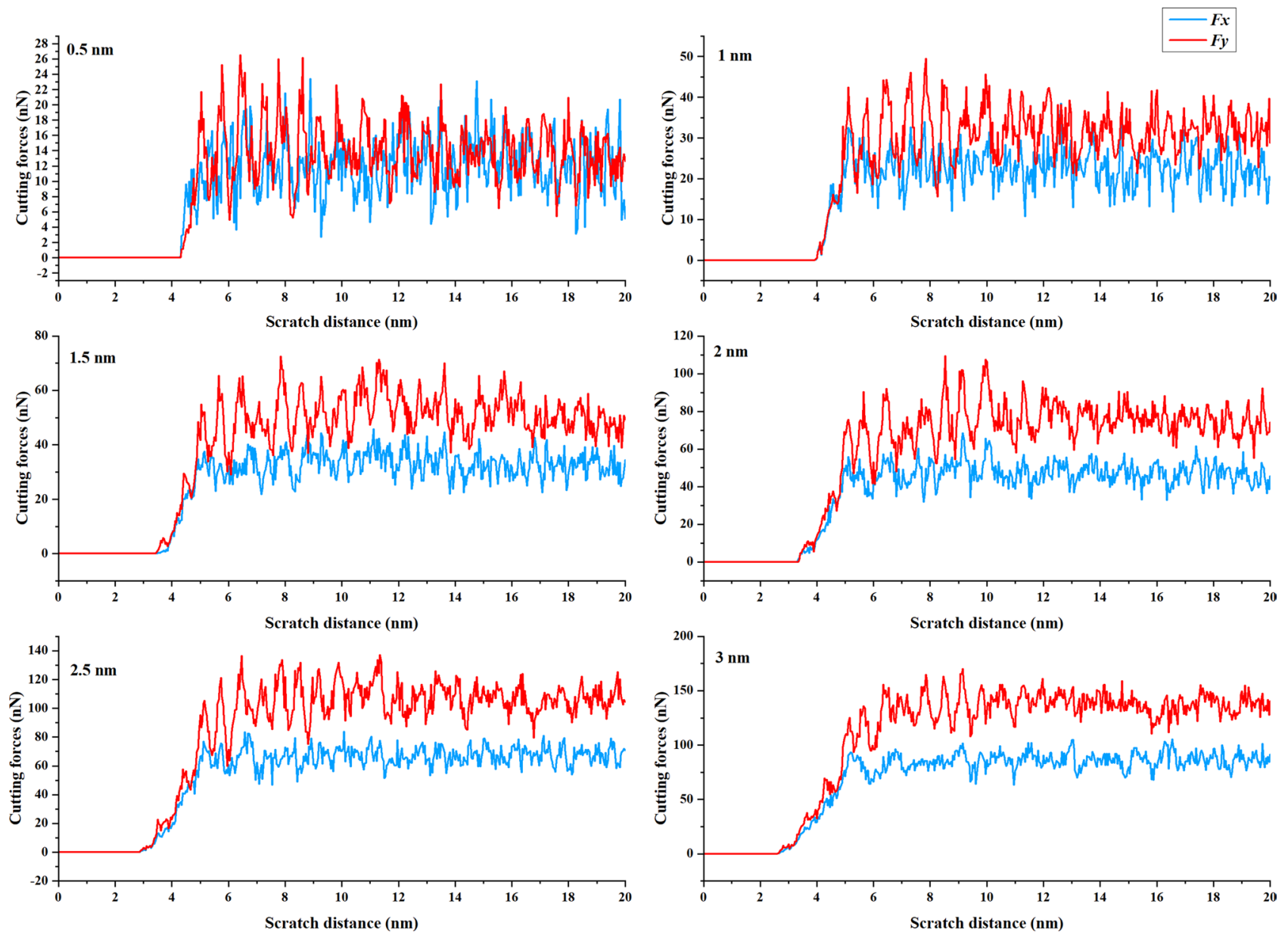

Fig. 5 Evolution of the $F x$ and $F y$ at scratch depths of $0.5,1,1.5,2,2.5$, and $3 \mathrm{~nm}$ plotted with respect to the simulation time steps corresponding to a total scratch distance of $20 \mathrm{~nm}$

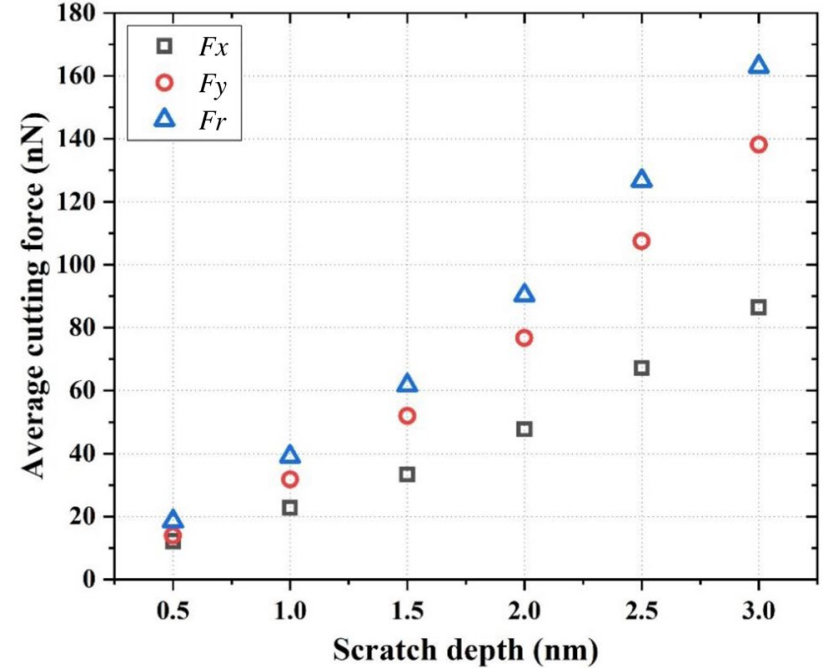

Fig. 6 Variations in the cutting forces obtained from the MD simulations with Fr as the resultant force

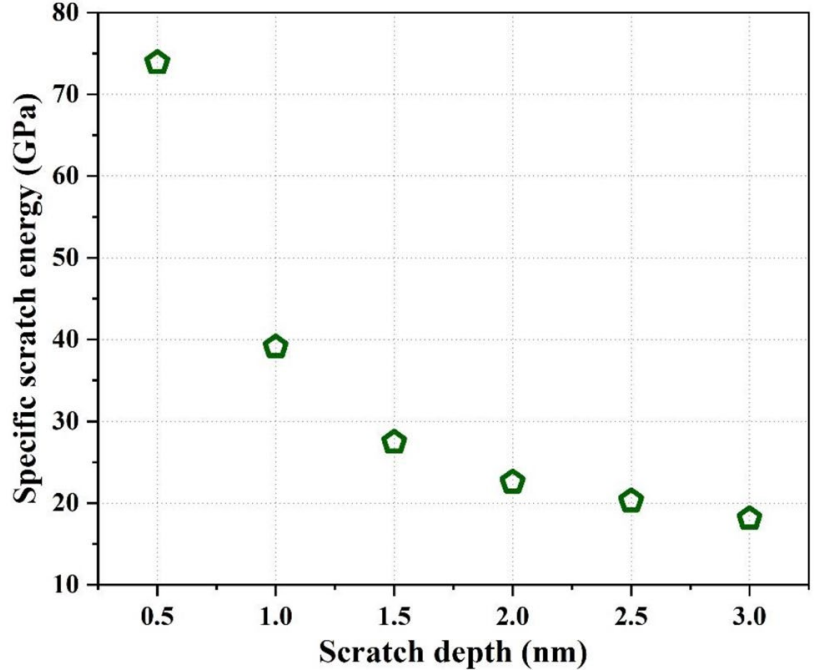

Fig. 7 Variation in the specific scratch energy for different scratch depth cases obtained from the MD simulations 


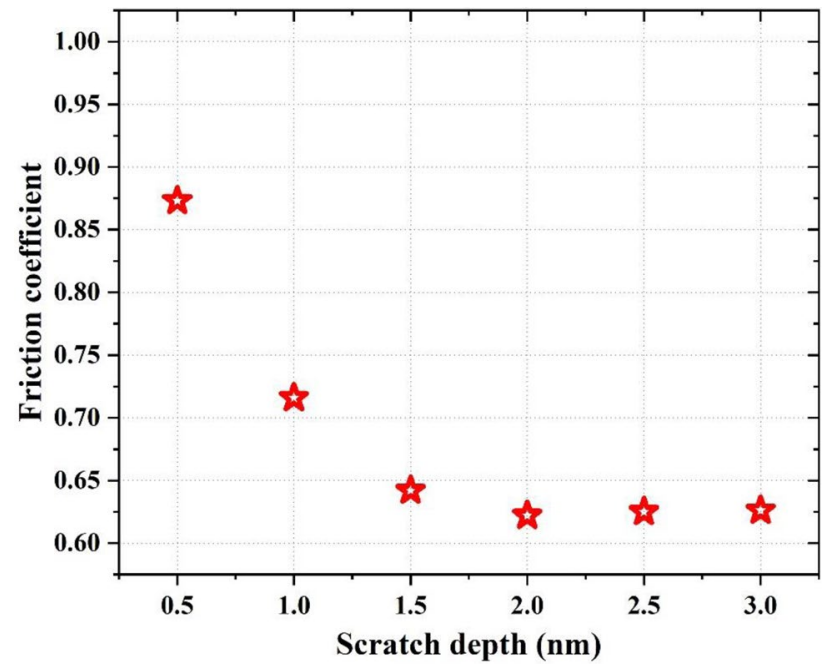

Fig. 8 Variation in the COF for different scratch depth cases obtained from the MD simulations
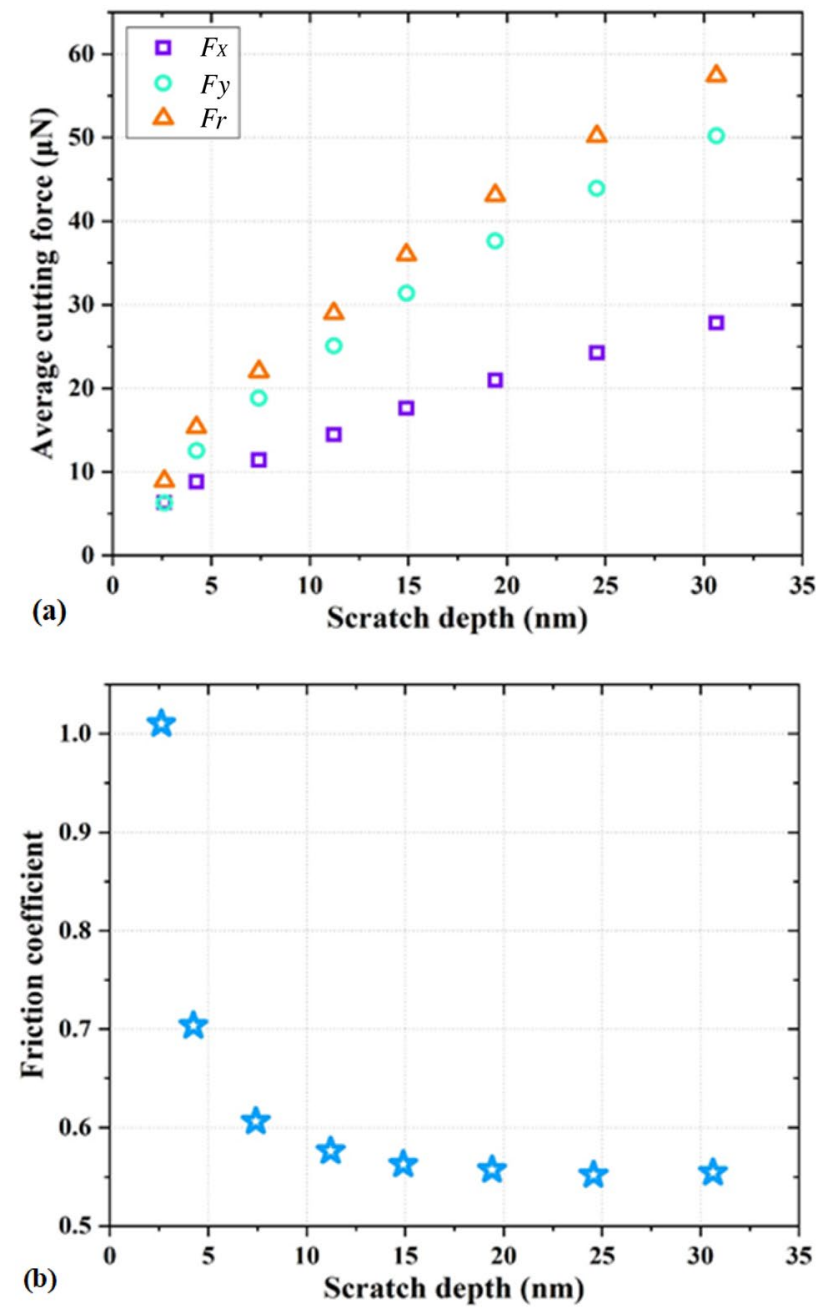

Fig. 9 Variation in the a scratch forces and $\mathbf{b}$ kinetic COF obtained from the AFM experiments of COF at a shallow scratch depth of $2 \mathrm{~nm}$ was approximately close to unity, but it reduced to almost a value of 0.55 at a higher scratch depth of $>15 \mathrm{~nm}$. This finding also indicated the influence of the included angle and the scratch depth. A sharper AFM tip can be expected to achieve lower COF at depths below $15 \mathrm{~nm}$, which was the case in the MD simulation, where the threshold of the size effect occurred at about $2 \mathrm{~nm}$.

\subsection{Flow Stress and Plasticity in GaAs}

A previous experimental work on doped GaAs material indicated the possibility that the phase transition from a zincblende structure (GaAs-I) to a rocksalt structure (GaAs-II) is responsible for the incipient plasticity during its nanoindentation process [38]. Without offering any direct evidence of the phase transition and at pressures as high as $<12 \mathrm{GPa}$, the work suggested that the GaAs-I to GaAs-II transition causes the plasticity in the material. During the present investigation, we used stress tensors to obtain the estimated von Mises stress in the cutting zone of the GaAs workpiece by using the same methodology that was previously used [39]. The von Mises stress we obtained in the cutting zone was around $7 \mathrm{GPa}$, which is closer to the value of the nanoindentation hardness of the GaAs material [40, 41], as illustrated in Fig. 10. The indentation hardness is representative of the plastic property of the material and coincides with the value of flow stress obtained in this work from the MD simulation, which possibly suggests that GaAs can flow plastically at a lower stress value than previously reported [38]. Moreover, the stress that is required to cause a phase transformation from GaAs-I to GaAs-II phase is of the order of $17.3 \mathrm{GPa}$, which is unlikely to occur during

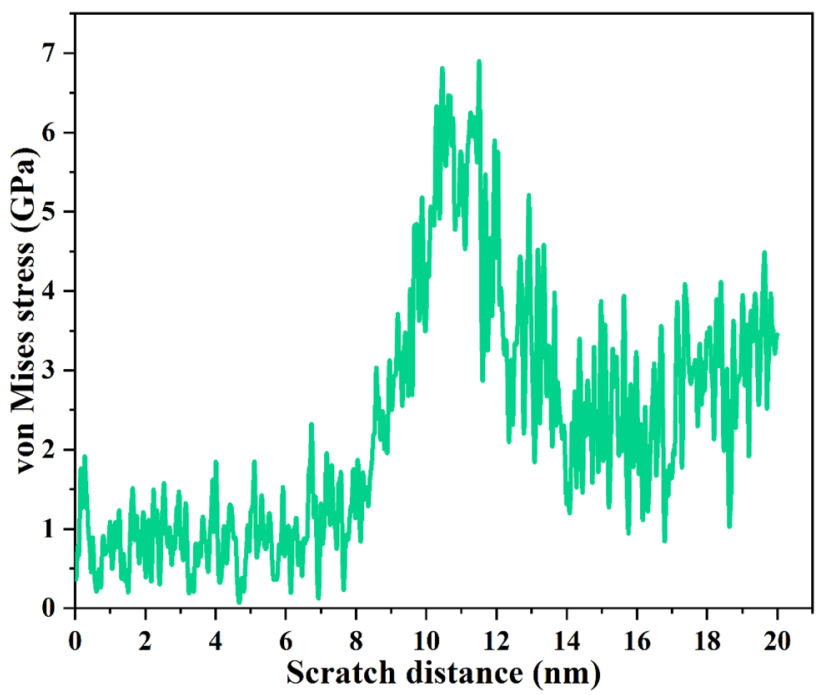

Fig. 10 Variation in von Mises stress at a scratch depth of $3 \mathrm{~nm}$ 
nanoscratching [42-44]. Hence, the phase transformation of GaAs during nanoscratching can be ruled out.

Furthermore, a Dislocation Extraction Algorithm [45] (DXA) was used to capture an instance of the emission of two dislocations in the subsurface belonging to the Burgers vector family of $1 / 2<110\rangle$, as shown in Fig. 11. This idea explains that the nucleation of dislocation can occur much earlier than the phase transformation and hence is energetically more favorable.

Overall, if the potential function used in this investigation (Albe type) is to be trusted, then the dislocation nucleation occurring at a lower stress value of $7 \mathrm{GPa}$, which coincides with the value of nanoindentation hardness (which is also a plastic property), seems to suggest that the primary reason plasticity occurs in GaAs is dislocation nucleation. This finding challenges existing studies reporting that phase transition is responsible for the ductile plasticity in GaAs.

\section{Remarks on Comparing MD Simulations with Experiments}

An important detail to recognize is that, while a very good qualitative comparison between the MD simulations and experiments is obtained in this investigation, differences exist in the situations; these differences cannot be neglected, and they require further discussions. Experimental techniques involving scanning probe microscopes such as AFM and STM have large dependencies on the stiffness of the system (cantilever/tip), whereas the MD simulation assumes an infinitely rigid diamond tip. Moreover, the experimental results can be influenced easily by extrinsic factors, such as surface contaminants, surface state, the geometry of the slider, tilting of the substrate or the tip, impurities in the substrate, and contact conditions at the interface. Also, the cutting velocity employed during the AFM scratches was on the order of few microns per second $(5 \mu \mathrm{m} / \mathrm{s})$ as opposed to the velocity of $10 \mathrm{~m} / \mathrm{s}$ used during the simulations. In view of this situation, the contact conditions during experiments were somewhat close to being quasistatic but were more dynamic during the simulations, thereby pointing us to strong strain rate effects in the MD. However, this condition should not affect the hardness, flow stress, and elastic modulus usually obtained from the MD simulation studies.

A higher cutting and/or sliding velocity needed to be employed to keep the computational requirements of the study within acceptable limits. Therefore, appropriate caution should be exercised in data interpretation. As such, the surface of the GaAs modeled in MD is atomically smooth and completely free of any contaminant, and the entire scratching process took place in a vacuum. With all these differences considered in the background, Fig. 12 shows a comparison of the kinetic $\mathrm{COF}$ and the specific scratching energy for the range of depth that is investigated commonly between MD and the AFM experiments.

Figure 12 reveals that the comparison of the specific scratching energy showed good proximity between the MD simulation and experiments, whereas the value of the kinetic $\mathrm{COF}$ exhibited differences. This finding indicates that the specific scratching energy could be insensitive to the velocity and the geometry of the indenter, but the kinetic COF is perhaps governed by certain other factors that need to be further investigated. In particular, the force ratio was substantially different at a very narrow scratch depth of $<2 \mathrm{~nm}$, which leads us to consider and plan follow-up work to investigate if this situation is due to previous surface amorphization, roughness, or the presence of an oxide layer as opposed to pure GaAs scratched in MD.
Fig. 11 Observation of subsurface at a scratch depth of $3 \mathrm{~nm}$

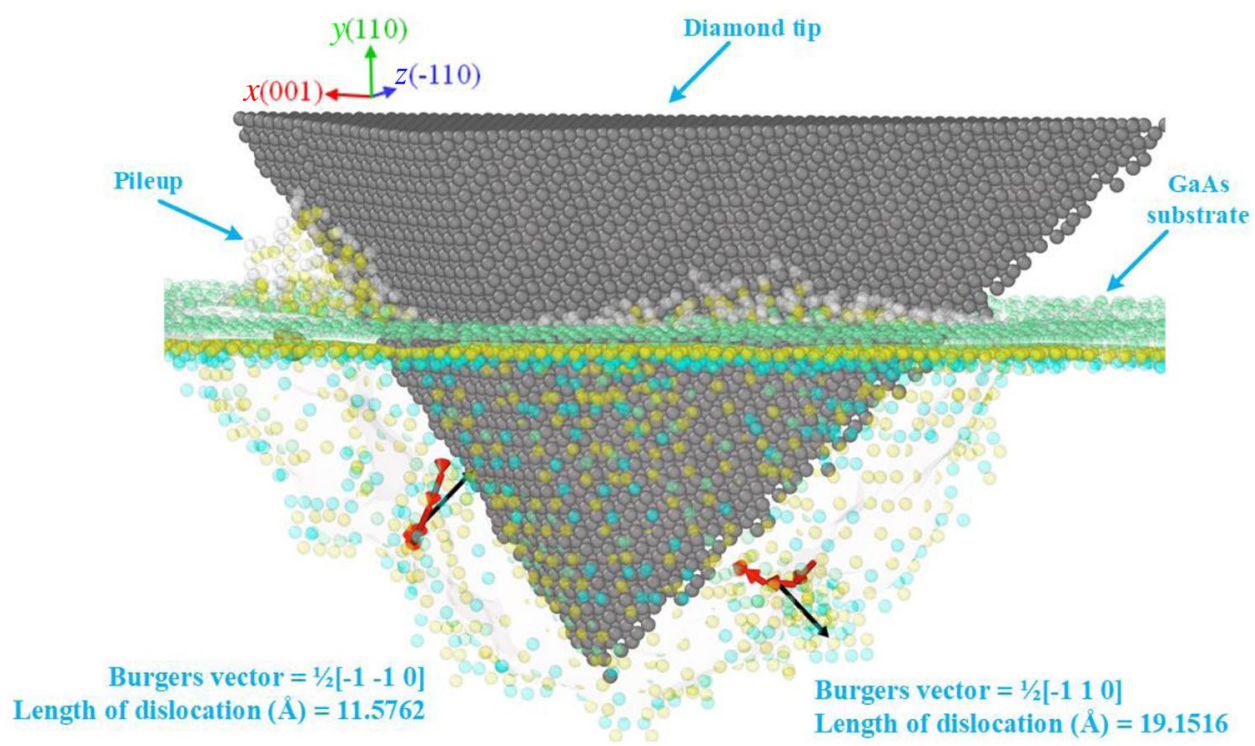



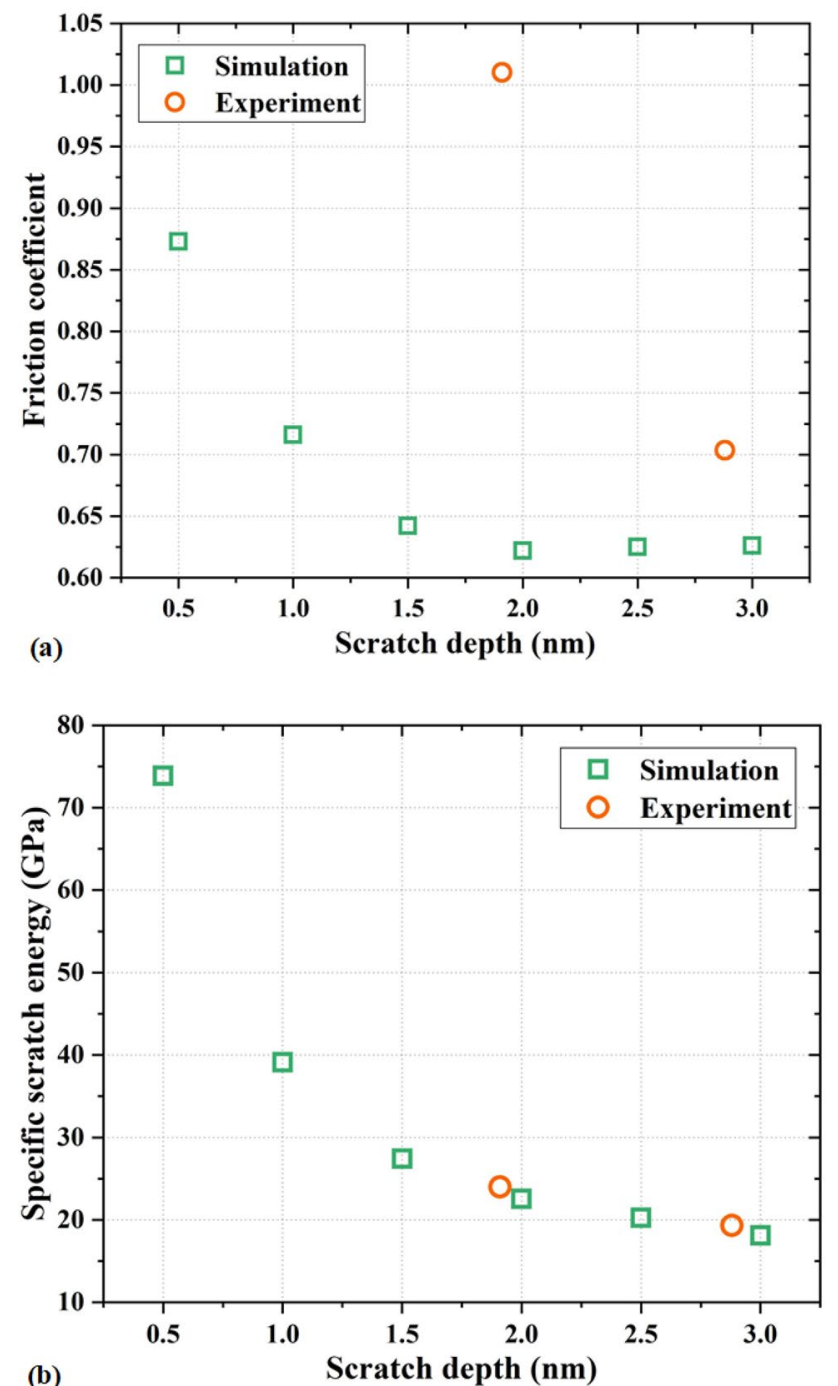

(b)

Fig. 12 Comparison of the MD simulation and experimental results a kinetic $\mathrm{COF}$ and $\mathbf{b}$ specific scratching energy

\section{Conclusions}

This work employed MD simulations and AFM scratch experiments to investigate the nanoscale friction and behavior of material removal on single-crystal(110)-oriented GaAs material surface. The following conclusions can be drawn from the aforementioned discussions:

1. A strong size effect was observed during GaAs nanoscratching at depths below $2 \mathrm{~nm}$ in the MD simulations and at depths below $15 \mathrm{~nm}$ in the AFM experiments. The tip sharpness used in the AFM was much higher than that used in MD. Thus, the size effect observed during nanoscratching can be concluded to be dependent on the geometry of the tip and the scratch depth.
2. During steady-state scratching, when the scratching is performed in a size effect-free regime, the kinetic $\mathrm{COF}$ revealed by the experiments and MD simulations was 0.55 and 0.62 , respectively. In both cases, the kinetic COF increased to a value of unity when scratching was performed in the size effect regime.

3. Likewise, a size effect was observed in the specific scratch energy because the value obtained while the (110) surface of GaAs was being scratched was about $20 \mathrm{GPa}$ in the size effect-free regime, but it went up to $90 \mathrm{GPa}$ at shallow scratch depths, thereby indicating that the workpiece has strong resistance to plastic deformation at narrower scratch depths.

4. An interesting observation was that the specific scratch energy was insensitive to the scratch speed and geometry of the tip, and good proximity was obtained between the MD simulations and experiments, whereas the kinetic COF showed some variations, albeit reasonably smaller ones. The specific scratch energy is inferred to be a more reliable indicator than the COF to validate the simulation studies where differences in speed and geometry are involved.

5. Contrary to the published experimental results that suggest a phase transformation from GaAs-I to GaAs-II (zincblende to rocksalt phase), which can occur at stress values $<13 \mathrm{GPa}$ only, the simulations performed in this work by using $\mathrm{ABOP}$ suggest that the von Mises stress values or the nanoindentation hardness can reach up to $7 \mathrm{GPa}$ only. At this lower stress value, phase transformation cannot take place. Instead, the incipient plasticity in single-crystal GaAs during nanoscratching occurred due to the $1 / 2<110>$-type dislocation nucleated in the subsurface of the GaAs.

Acknowledgements The authors would like to thank EPSRC (EP/ K018345/1, EP/T024844/1) and the Royal Society-NSFC International Exchange Program (IECINSFC 181474) for providing financial support to this research. SG is particularly thankful for the research support provided by the UKRI via Grant Nos. EP/L016567/1, EP/ S013652/1, EP/T001100/1, EP/S036180/1, and EP/T024607/1. The authors also acknowledge the support received from H2020 (Cost Actions CA18125, CA18224, CA17136, and CA16235); the Royal Academy of Engineering via Grant Nos. IAPP18-19\295, TSP1332, and EXPP2021\1\277; and the Newton Fellowship award from the Royal Society (NIF\R1\191571). SG also accessed the Isambard Bristol, a UK supercomputing service, via the Resource Allocation Panel and ARCHER2 resources (Project e648). The authors also acknowledge the use of the EPSRC (EP/K000586/1)-funded ARCHIE-WeSt HighPerformance Computer at the University of Strathclyde.

Data Availability All data underpinning this publication are openly available from the University of Strathclyde Knowledge Base at https:// doi.org/10.15129/7e090adb-7c67-41c5-aaf2-bc897caffe2d. 


\section{Declaration}

Conflict of interest The authors declare that they have no conflicts of interest.

Open Access This article is licensed under a Creative Commons Attribution 4.0 International License, which permits use, sharing, adaptation, distribution and reproduction in any medium or format, as long as you give appropriate credit to the original author(s) and the source, provide a link to the Creative Commons licence, and indicate if changes were made. The images or other third party material in this article are included in the article's Creative Commons licence, unless indicated otherwise in a credit line to the material. If material is not included in the article's Creative Commons licence and your intended use is not permitted by statutory regulation or exceeds the permitted use, you will need to obtain permission directly from the copyright holder. To view a copy of this licence, visit http://creativecommons.org/licenses/by/4.0/.

\section{References}

1. Altuntas H, Ozcelik S (2013) The interface states and series resistance analyzing of $\mathrm{Au} / \mathrm{SiO}_{2} / \mathrm{n}-\mathrm{GaAs}$ at high temperatures. J Alloys Compd 577:143-147

2. Martelli F, Priante G, Rubini S (2015) Photoluminescence of GaAs nanowires at an energy larger than the zincblende bandgap: dependence on growth parameters. Semicond Sci Technol 30(5): $1-6$

3. Song Y, Zhang X, Yan X, Liao Q, Wang Z, Zhang Y (2014) An enzymatic biosensor based on three-dimensional $\mathrm{ZnO}$ nanotetrapods spatial net modified AlGaAs/GaAs high electron mobility transistors. Appl Phys Lett 105(21):213703

4. Hyon CK, Choi SC, Hwang SW, Ahn D, Kim Y, Kim EK (1999) Direct nanometer-scale patterning by the cantilever oscillation of an atomic force microscope. Appl Phys Lett 75(2):292-294

5. Versen M, Klehn B, Kunze U, Reuter D, Wieck AD (2000) Nanoscale devices fabricated by direct machining of GaAs with an atomic force microscope. Ultramicroscopy 82(1-4):159-163

6. Komanduri R, Chandrasekaran N (2000) Molecular dynamics simulations of atomic-scale friction. Phys. Rev. B 61(20):167-172

7. Goel S, Stukowski A, Luo X, Agrawal A, Reuben RL (2013) Anisotropy of single-crystal 3C-SiC during nanometric cutting. Modell Simul Mater Sci Eng 21(6):065004

8. Qu J, Blau PJ, Watkins TR, Cavin OB, Kulkarni NS (2005) Friction and wear of titanium alloys sliding against metal, polymer, and ceramic counterfaces. Wear 258(9):1348-1356

9. Butt MZ, Khaleeq-Ur-Rahman M, Ali D (2010) Kinetics of flow stress in ultra-pure tantalum single crystals in stress/temperature regime III. J Mater Sci 45(22):6046-6051

10. Prasad SV, Michael JR, Battaile CC, Majumdar BS, Kotula PG (2020) Tribology of single crystal nickel: interplay of crystallography, microstructural evolution, and friction. Wear 458-459:203320

11. Takagi R, Tsuya Y (1961) Static friction between clean copper single-crystal surfaces. Wear 4(3):216-227

12. Gu D, Zhang L, Chen S, Song K, Liu S (2018) Significant reduction of the friction and wear of PMMA-based composite by filling with PTFE. Polymers (Basel) 10(9):966

13. Liu HT, Zhao MH, Lu C, Zhang JW (2020) Characterization on the yield stress and interfacial coefficient of friction of glasses from scratch tests. Ceram Int 46(5):6060-6066

14. Zhang Q, Diao D, Kubo M (2015) Nanoscratching of multi-layer graphene by molecular dynamics simulations. Tribol Int $88: 85-88$
15. Chen C, Lai M, Fang F (2021) Study on the crack formation mechanism in nano-cutting of gallium arsenide. Appl. Surf. Sci. 540(P2): 148322

16. Chen YH, Huang H, Lu MY, Wu YQ, Fang FZ, Hu XT (2014) Molecular dynamics simulation of the deformation of singlecrystal gallium arsenide. Appl Mech Mater 553:60-65

17. Yi D, Li J, Zhu P (2018) Study of nanoscratching process of GaAs using molecular dynamics. Curr Comput Aided Drug Des 8(8):321

18. Plimpton SJ (1995) Fast parallel algorithms for short range molecular dynamics. J Comput Phys 117(1):1-19

19. Fan P, Ding F, Luo X, Yan Y, Geng Y, Wang Y (2020) A simulated investigation of ductile response of GaAs in single-point diamond turning and experimental validation. Nanomanuf Metrol 3(4):239-250

20. Goel S, Luo X, Agrawal A, Reuben RL (2015) Diamond machining of silicon: a review of advances in molecular dynamics simulation. Int J Mach Tool Manuf 88:131-164

21. Xiao G, He Y, Geng Y, Yan Y, Ren M (2019) Molecular dynamics and experimental study on comparison between static and dynamic ploughing lithography of single-crystal copper. Appl Surf Sci 463(August):96-104

22. Yan Y, He Y, Xiao G, Geng Y, Ren M (2019) Effects of diamond tip orientation on the dynamic ploughing lithography of singlecrystal copper. Precis Eng 57:127-136

23. Berendsen HJC, Postma JPM, Van Gunsteren WF, Dinola A, Haak JR (1984) Molecular dynamics with coupling to an external bath. J Chem Phys 81(8):3684-3690

24. Fan P, Goel S, Luo X, Yan Y, Geng Y, Wang Y (2021) An atomistic investigation on the wear of diamond during atomic force microscope tip-based nanomachining of gallium arsenide. Comput Mater Sci 187:110115

25. Nosé $S$ (1984) A unified formulation of the constant temperature molecular-dynamics methods. J Chem Phys 81(1):511-519

26. Chavoshi SZ, Xu S, Goel S (2017) Addressing the discrepancy of finding the equilibrium melting point of silicon using molecular dynamics simulations. Proc R Soc A Math Phys Eng Sci 473(2202):1-9

27. Murdick DA, Zhou XW, Wadley HNG, Nguyen-Manh D, Drautz R, Pettifor DG (2006) Analytic bond-order potential for the gallium arsenide system. Phys Rev B 73(4):1-20

28. Ward DK, Zhou XW, Wong BM, Doty FP, Zimmerman JA (2012) Analytical bond-order potential for the cadmium telluride binary system. Phys Rev B 85(11):1-19

29. Ziegler JF, Ziegler MD, Biersack JP (2010) SRIM - The stopping and range of ions in matter (2010). Nucl Instrum Methods Phys Res B 268(11-12):1818-1823

30. https://lammps.sandia.gov/doc/pair_zbl.html. Accessed on $11 / 5 / 2021$

31. Stukowski A (2010) Visualization and analysis of atomistic simulation data with OVITO-the Open Visualization Tool. Modell Simul Mater Sci Eng 18(1):015012

32. Goel $\mathrm{S}$ et al (2020) Horizons of modern molecular dynamics simulation in digitalized solid freeform fabrication with advanced materials. Mater Today Chem 18:100356

33. Al-Musawi RSJ, Brousseau EB, Geng Y, Borodich FM (2016) Insight into mechanics of AFM tip-based nanomachining: Bending of cantilevers and machined grooves. Nanotechnology 27(38):385302

34. Goel S, Martinez FD, Chavoshi SZ, Khatri N, Giusca C (2018) Molecular dynamics simulation of the elliptical vibration-assisted machining of pure iron. J Micromanuf 1(1):6-19

35. Goel S, Luo X, Reuben RL (2013) Wear mechanism of diamond tools against single crystal silicon in single-point diamond turning process. Tribol Int 57:272-281 
36. Goel S (2014) The current understanding on the diamond machining of silicon carbide. J. Phys. D Appl. Phys 47(24):243001

37. Pan Y et al (2021) New insights into the methods for predicting ground surface roughness in the age of digitalisation. Precis Eng 67(October):393-418

38. Chrobak D, Trębala M, Chrobak A, Nowak R (2019) Origin of nanoscale incipient plasticity in GaAs and InP crystal. Crystals 9(12):651

39. Goel S, Kovalchenko A, Stukowski A, Cross G (2016) Influence of microstructure on the cutting behaviour of silicon. Acta Mater 105:464-478

40. Ericson F, Johansson S, Schweitz JÅ (1988) Hardness and fracture toughness of semiconducting materials studied by indentation and erosion techniques. Mater Sci Eng 105-106(1):131-141
41. Fang TH, Chang WJ, Lin CM (2005) Nanoindentation and nanoscratch characteristics of $\mathrm{Si}$ and GaAs. Microelectron Eng 77(3-4):389-398

42. Ono S, Kikegawa T (2018) Phase transformation of GaAs at high pressures and temperatures. J Phys Chem Solids 113:1-4

43. Besson JM, Itié JP, Polian A, Weill G, Mansot JL, Gonzalez J (1991) High-pressure phase transition and phase diagram of gallium arsenide. Phys Rev B Condens Matter 44(9):4214-4234

44. Ovsyannikov SV, Shchennikov VV (2006) Observation of a new high-pressure semimetal phase of GaAs from pressure dependence of the thermopower. J Phys Condens Matter 18(42):L551-L557

45. Stukowski A, Bulatov VV, Arsenlis A (2012) Automated identification and indexing of dislocations in crystal interfaces. Modell Simul Mater Sci Eng 20(8):085007 